\title{
The Payne effect revisited
}

\author{
R. Hentschke* \\ School of Mathematics and Natural Sciences, Bergische Universität, D-42097 Wuppertal, Germany
}

Received 22 September 2016; accepted in revised form 24 November 2016

\begin{abstract}
Measurements of the storage modulus, $\mu^{\prime}$, vs. strain amplitude, $u$, for highly filled rubbers exhibit a pronounced decrease of $\mu^{\prime}$ with increasing $u$. Unfilled rubbers do not show this so called Payne effect. Even though the effect is known since the 1940s, it continues to play a significant role in the research community focusing on rubber materials in general and automobile tires in particular. The key problem is the elucidation of the dependence of the Payne effect on the chemical composition of the rubber material. Based on a scaling approach we derive the functional form $\mu^{\prime}(u, T)-\mu^{\prime}(\infty, T) \propto$ $(1-(D / d) u)^{-\left(\sigma-d_{\mathrm{f}}+1\right)} g(T)$, where the parameters $D / d$ and $\sigma-d_{\mathrm{f}}+1$ are directly related to the filler network structure. In addition, we explain the temperature dependence of the Payne effect, $g(T)$, in terms of a distribution of activation energies corresponding to different types of filler-filler interactions. Finally, the model is extended to describe the attendant amplitude dependence of the loss modulus.
\end{abstract}

Keywords: rubber, nano-composite, reinforcement, mechanical properties, modelling

\section{Introduction}

The dynamic moduli of filled elastomers do depend on strain amplitude, strain frequency or temperature as well as the history thereof and, in particular, on the composition of the elastomer including its processing conditions. One of the key effects in this context is the Payne effect, i.e. the pronounced decrease of the storage modulus with increasing strain amplitude in filled rubbers under cyclic loading. The effect, already observed by a number of researchers in the early 1940s [1], was studied and described extensively by Payne in the 1960s (an early review is reference [2]; more recent reviews are references [35]). Because the Payne effect does not occur in unfilled rubbers, its cause must be related to either the rubber-filler or the filler-filler interface(s).

A number of explanations and attendant models for the Payne effect can be found in the literature. These include filler network breakdown [2, 6] (chapter 3) [7-9] filler deagglomeration in the framework of selfsimilarity $[10,11]$, polymer debonding from filler surface $[12,13]$, strain softening of the polymer shell surrounding fillers [14], a network junction model at small strain [3, 4], or a recent approach based statistical mechanics in the context of the jamming transition [15]. Another type of approach is taken in certain phenomenological material models on the basis of visco-mechanical elements or networks thereof and their mathematical exploitation (e.g. [16-20]). The distinct advantage of this general approach is the inclusion of the frequency dependence and relaxation as well as history effects in addition to amplitude dependence. Nonetheless it is probably fair to say that there continues to be a lack of interrelation between the chemical composition of the rubber material, molecular mechanisms and the macroscopic moduli. If for instance the polymer or a coupling agent is modified through certain functional groups or altered in its molecular architecture, it is desirable to predict the effect on the latter on the dynamic moduli and perhaps on performance parameters like the rolling resistance. 
In this work we discuss both the amplitude, applying the concept of self-similarity (cf. [10, 11]), as well as the temperature dependence of the Payne effect. We develop a new model replacing the standard form $\mu^{\prime}(u)-\mu^{\prime}(\infty) \propto\left(1-\left(u / u_{0}\right)^{2 \mathrm{~m}}\right)^{-1}$, with a modified expression, i.e. $\mu^{\prime}(u)-\mu^{\prime}(\infty) \propto(1-(D / d) u)^{-\left(\sigma-\mathrm{d}_{\mathrm{f}}+1\right)}$, where the parameters $D / d$ and $\sigma-d_{\mathrm{f}}+1$ are directly related to filler properties and network structure. We also explain the temperature dependence of the Payne effect in terms of a distribution of activation energies offering new insight regarding the connection between surface modification of fillers, silica in particular, and attendant effects on $\mu^{\prime}=\mu^{\prime}(u, T)$. Finally, we discus the application of these ideas to the calculation of the amplitude dependence of the loss modulus.

The paper is structured as follows. We begin with a brief review of the Maier and Göritz model, which is built on the idea of chain-slippage, a loss mechanism frequently used to interpret experimental data. In addition, the resulting expression for the amplitude dependence of the Payne effect provides the functional form commonly used to fit attendant measurements. After a brief discussion of what we consider the conceptual weaknesses of this approach, we develop an alternative model based on self-similarity of the filler network. Subsequently we address the temperature dependence of the Payne effect, which is followed by a discussion of a number of experimental key observations and their explanation by the model. In the second to last section the new model is applied to the calculation of the strain dependence of the loss modulus. The last section is the conclusion.

\section{Amplitude dependence: The model of Maier and Göritz}

Maier and Göritz [12] have developed a simple phenomenological model of the Payne effect, which is motivated by the idea of chain-slippage at the fillerrubber interface. In addition, the mathematical form of their final expressions for the amplitude dependence of the dynamic moduli is quite similar to the attendant expressions derived by Kraus [6, 7] or Huber and Vilgis [11].

Maier and Göritz consider a two-state equilibrium. In state $\mathrm{A}$ a short polymer segment interacts strongly with the surface (physisorbed segment); in state B the same segment interacts weakly, because it has become detached. We introduce the corresponding state mole fractions $x_{\mathrm{A}}$ and $x_{\mathrm{B}}$, i.e. $x_{\mathrm{A}}+x_{\mathrm{B}}=1 . x_{\mathrm{A}}$ and $x_{\mathrm{B}}$ are not constant. It is assumed that the following balance Equation (1) is satisfied:

$x_{\mathrm{A}} p_{\mathrm{A} \rightarrow \mathrm{B}}=x_{\mathrm{B}} p_{\mathrm{B} \rightarrow \mathrm{A}}$

where $p_{\mathrm{A} \rightarrow \mathrm{B}}$ and $p_{\mathrm{B} \rightarrow \mathrm{A}}$ are equilibrium state-to-state transition probabilities. Hence Equation (2) follows:

$x_{\mathrm{A}}=(1+q)^{-1}$

where $q=p_{\mathrm{A} \rightarrow \mathrm{B}} / p_{\mathrm{B} \rightarrow \mathrm{A}}$. At this point Maier and Göritz assume Equation (3):

$q=\left(\frac{u}{u_{0}}\right)^{2 \mathrm{~m}}$

The quantity $u$ is the dynamic strain amplitude. $u_{0}$ and $m$ are parameters. In the above reference [12] $2 m=1$. If the amplitude dependent part of the storage modulus, $\Delta \mu^{\prime}(u)$, is proportional to $x_{\mathrm{A}}$, then Equation (4) follows:

$\Delta \mu^{\prime}(u)=\frac{\Delta \mu^{\prime}(0)}{1+\left(\frac{u}{u_{0}}\right)^{2 \mathrm{~m}}}$

A corresponding expression for the loss modulus can be obtained if the latter is assumed to be proportional to $x_{\mathrm{A}} x_{\mathrm{B}}$. It is common to plot $\Delta \mu^{\prime}(u)$ vs. $\ln _{10} u$, i.e. if we define $\tilde{\mathrm{u}}=\ln _{10} u$ then we obtain Equation (5):

$\Delta \mu^{\prime}(u)=\frac{\Delta \mu^{\prime}(0)}{1+10^{\left[2 \mathrm{~m}\left(\tilde{\mathrm{u}}-\tilde{\mathrm{u}}_{0}\right)\right]}}$

In this case the curve has an inflection point at $\tilde{u}=u_{0}$. Its slope at this point is $-m(\ln [10] / 2) \Delta \mu^{\prime}(0)$. Formula (5) usually fits the measured $\Delta \mu^{\prime}(u)$ quite well. The combination of simplicity and being a good fit function for $\Delta \mu^{\prime}(u)$-data makes the model attractive. The attractiveness is enhanced by the fact that the attendant expression for the loss modulus $\Delta \mu^{\prime \prime}(u) \propto$ $x_{\mathrm{A}} x_{\mathrm{B}}=x_{\mathrm{A}}\left(1-x_{\mathrm{A}}\right)$ is also qualitatively correct (except at small amplitudes). But this may be deceiving.

Equation (3) is a mere assumption without theoretical backing (the same is true for $\Delta \mu^{\prime \prime}(u) \propto x_{\mathrm{A}} x_{\mathrm{B}}$. This brings about the next point. Equation (1) describes a molecular equilibrium, which depends on temperature, $T$. From equilibrium thermodynamics we expect $x_{\mathrm{A}}=x_{\mathrm{A}}(T)$, with an exponential temperature dependence (cf. below). This in turn means that according to the assumption (3) the strain amplitude should be related to temperature akin to the frequency in the well 
known time-temperature-superposition principle, i.e. $u=u(T)$. Hence, $\mu^{\prime}\left(u_{\text {large }}, T_{\text {small }}\right)$ should directly correspond to some $\mu^{\prime}\left(u_{\text {small }}, T_{\text {large }}\right)$. While a correspondence between strain and temperature is conceptually reasonable in the context of the above 'chain segment slippage'-mechanism, it is not possible to find an attendant 'shift factor' allowing to map out $\mu\left(u, T_{\text {large }}\right)$ on the basis of $\mu^{\prime}\left(u, T_{\text {small }}\right)$ over the entire range of amplitudes. Less severe but also bothersome is that $u_{0}$ in the above model is a mere fit parameter, with no particular tie to molecular processes underlying the Payne effect. In the case of the exponent $m$ the situation is somewhat different. $m$ has been related to two other exponents, $d_{\mathrm{f}}$, the mass fractal dimension of the filler network, and $C$, the scaling dimension of the minimum path (i.e. $L \sim R^{\mathrm{C}}$, where in this case $L$ is the minimum path length between two points separated by the distance $R$ in a fractal), by $m^{-1}=C-d_{\mathrm{f}}+2$ (e.g. [5]). The value quoted in this source is $m \approx 0.66$. Indeed in many cases a fit to experimental data for $\Delta \mu^{\prime}(u)$, using this value while adjusting the other parameters, produces good agreement. However, $m \approx 0.66$ is based on certain models of cluster-cluster-aggregation yielding $d_{\mathrm{f}} \approx 1.8$. A recent SAXS (Small Angle X-ray Scattering) study of silica filled rubbers finds a significantly larger value, i.e. $d_{\mathrm{f}} \approx 2.4$ [21]. Thus, even if the above scaling relation holds true, the problem then becomes the calculation of $C$ and $d_{\mathrm{f}}$ for different material composition. Generally, a variable $m$ is necessary in order for Equation (4) to be a good description of experimental $\mu^{\prime}(u)$.

\section{Amplitude dependence: Scaling model}

Figure 1 depicts a (model) filler distribution in an elastomer matrix. In practice the filler content is rather high and beyond the percolation threshold. The common picture, supported by TEM (Transmission Electron Microscopy) and SAXS experiments, describes the filler as composed of unbreakable aggregates of primary particles assembled into a superstructure of network-forming agglomerates [5, 22]. The agglomerate network can break under strain. The key elements in our model are paths connecting points (particles) in the network. The paths are characterised by their backbone length, $L$, and an attendant end-to-end distance, $R$. A certain number of these paths bear the load imposed by an attendant strain amplitude, $u$, due to external stress. When a

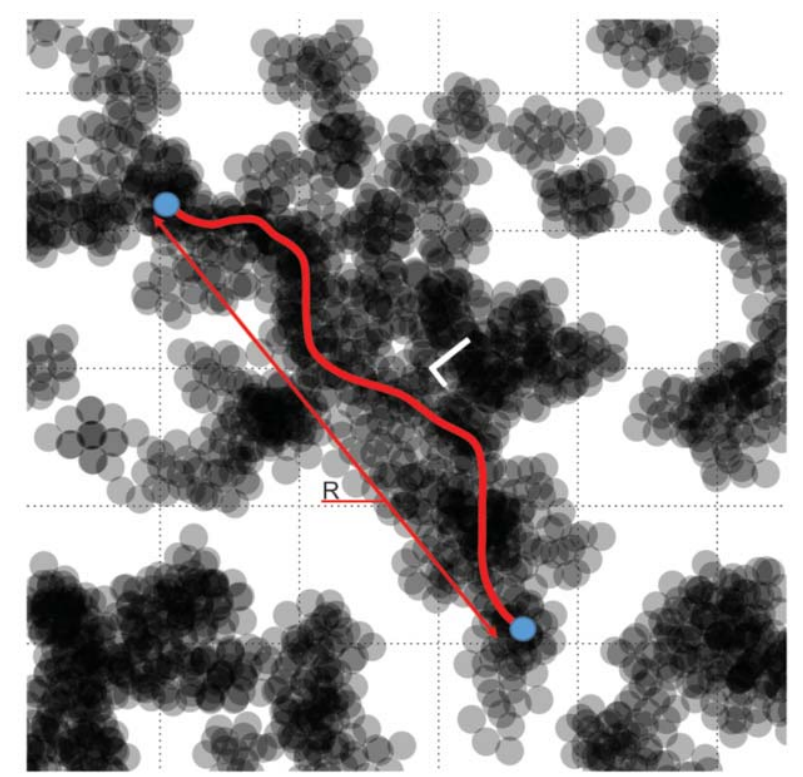

Figure 1. Filler distribution (black) inside an elastomer matrix (white). The red line indicates the backbone of a path.

load-bearing path breaks the load is redistributed and other paths will become load-bearing.

The ensemble of paths is characterised by a distribution function $H(L, R)$. However, we can expect that not all paths are equally important for the strength of a network at a given amplitude. In the following we therefore assume that the load-bearing paths are the so called minimum paths, characterised by the scaling relation $L \sim R^{\mathrm{C}}$, where $C$ is an attendant scaling exponent, connecting $L$ and $R$. Consequently we do not need $H(L, R)$. Instead we focus on $h(L)$, the distribution of minimum paths only. Notice in this context that it has been argued before that the network structure is self-similar (one important attendant result is the power law dependence of the storage modulus on filler volume fraction based on the concept of fractal flocs) (e.g. [5] and references therein). Clearly, there is a lower limit to self-similarity imposed by the particle size as well as an upper limit due to the mixing process. Underlying our entire reasoning is the assertion that it is the self-similar character of the network which governs the Payne effect. In this spirit we consider $h(L)$ to be a generalised homogeneous function, as shown by Equation (6):

$$
h(L)=\lambda h\left(L \lambda^{1 / y}\right)
$$

where $\lambda$ is an arbitrary parameter and $y$ is a number (e.g. reference [23]). We can apply this equation to 
a strained system where $L^{\prime}=(1+u) L$. Inserting this into (6) yields Equation (7):

$h\left(L^{\prime}\right)=\lambda h\left((1+u) L \lambda^{1 / \mathrm{y}}\right)$

If we chose $\lambda=((1+u) L)^{-\mathrm{y}}$ we find Equation (8):

$h\left(L^{\prime}\right)=\frac{1}{(1+u)^{\mathrm{y}}} L^{-\mathrm{y}} h(1)$

Because we already have assumed $\Delta \mu^{\prime}(u) \propto h\left(L^{\prime}\right)$, Equation (8) implies Equation (9):

$\Delta \mu^{\prime}(u) \sim \frac{1}{(1+u)^{y}}$

This is quite similar to Equation (4). The main difference is that here $u_{0}=1$. However, when Equation (4) is fitted to real data, the majority of $u_{0}$-values are in the range $0.02<u_{0}<0.2$, i.e. Equation (9) cannot be correct as is stands.

When a polymer chain is stretched the ratio $R / L$ increases due to the attendant conformation change without affecting molecular bonds or valence angles. The paths in the filler network are not polymers. They cannot alter their conformation easily and much of the external stress leads to strain in the gaps between aggregates along a path. We stress that the gaps are not empty. Depending on their width and the type of filler they may contain polymer, silanes, silanol groups, water molecules, etc. Figure 2 depicts a one-dimensional cartoon showing a line of aggregates of diameter $D$ separated by gaps of width $d$. The aggregates are rigid and thus the entire strain of the chain, $u$, is due to the strain in the gaps, as shown by Equation (10):

$u=\frac{d^{\prime}+D-(d+D)}{d+D} \approx \frac{d^{\prime}-d}{D}=\frac{d}{D} u_{\text {nano }}$

where $u_{\text {nano }}=\left(d^{\prime}-d\right) / d$. Therefore we must redefine the above path length $L$ via Equation (11):

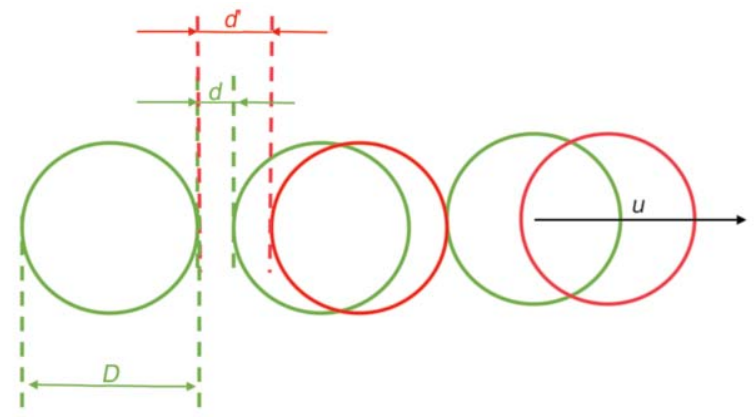

Figure 2. Cartoon of a linear path of aggregates at $u=0$ (green) and $u>0$ (red)
$L=\sum_{\mathrm{i}=1}^{\mathrm{N}} d_{\mathrm{i}}$

where $d_{\mathrm{i}}$ is the ith gap along a load-bearing path subject to strain and $N$ is the number of gaps in that path. Using this new definition of $L$ does not alter the general reasoning which led to Equation (9), but brings about the replacement of $u$ by $u_{\text {nano }}$. However, $u_{\text {nano }}$ is related to $u$ via Equation (10) and thus we obtain Equation (12):

$\Delta \mu^{\prime}(u) \sim \frac{1}{\left(1+\frac{D}{d} u\right)^{y}}$

This means that $u_{0} \equiv d / D$ in the present model, where the effective gap width, $d$, should be significantly smaller than the effective aggregate diameter, $D$.

As before in the case of Equation (4) we can introduce $\tilde{\mathrm{u}}=\ln _{10} u$, we obtain Equation (13):

$\Delta \mu^{\prime}(u)=\frac{\Delta \mu^{\prime}(0)}{\left(1+10^{\tilde{u}-\tilde{u}_{0}}\right)^{y}}$

where $\tilde{u}_{0}=\ln _{10}[d / D]$. This curve has its inflection point at $\tilde{u}=\tilde{u}_{0}-\ln _{10} y$, where the slope is $-((y+1) / y)^{-(\mathrm{y}+1)} \Delta \mu^{\prime}(0) \ln _{10}$.

But what is the physical meaning of the exponent $y$ ? In the case of a polymer $h(R L) \propto$ $(R L)^{\mathrm{d}-1} \exp \left[-(3 / 2) R^{2} /\left(L a^{2}\right)\right]$, where $d$ is the space dimension (not to be confused with the above quantity $d$ !) and a is a typical segment length (e.g. reference [24]). The exponential factor is due to the random walk character of the polymer, whereas the factor $(R / L)^{\mathrm{d}-1}$ is a property of space. We already emphasised that the load-bearing path are not polymers. But we should expect that the space-factor persists, albeit with $d$ replaced by the mass fractal exponent, $d_{\mathrm{f}}$, due to the assumed fractal geometry of the filler network on the here relevant spatial scale. The exponential factor, on the other hand, we replace by the power law $L^{-\sigma}$. We expect $\sigma>0$, because long paths should be less likely than short ones. Thus we have Equation (14):

$y=\sigma-d_{\mathrm{f}}+1$

Figure 3 shows a collection of experimental Payne curves for different systems from different sources. The solid lines are fits using Equation (12) expressed via Equation (13). The main parameters determining the shape of the curve are $D / d$ and $y$. Their numerical 


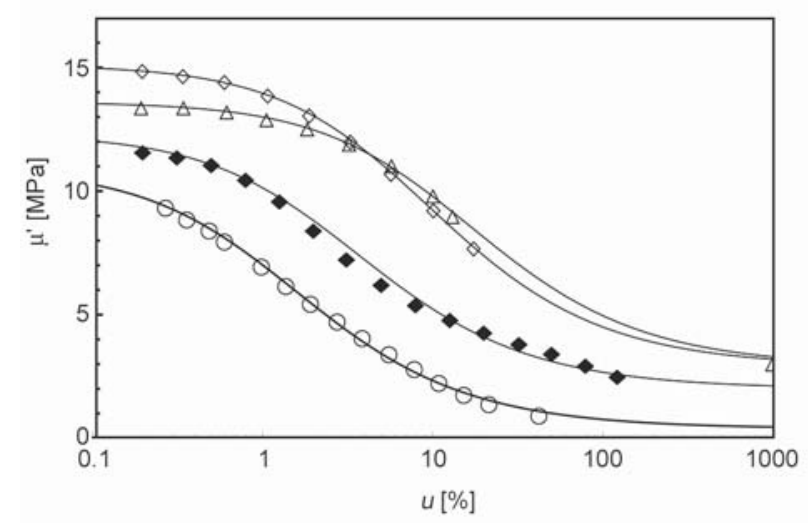

Figure 3. Fit of Equation (13) to measurements of the storage modulus from different sources. Open triangles $\left(\right.$ Aerosil $\left.^{\circledR} 200\right)$ and open diamonds (Aerosil ${ }^{\circledR} 200$ + TESPT): reference [25] (data taken at $10 \mathrm{~Hz}$ ); open circles: reference [26] (Figure 5.11, $T=$ $443 \mathrm{~K}$ ); solid diamonds: reference [27] (data taken at $10 \mathrm{~Hz}$ )

values corresponding to the different fits are compiled in Table 1. In addition we must fix the values of $\mu^{\prime}(0)$ as well as $\mu^{\prime}(\infty)$. Whereas the former usually is given be the experimental storage modulus at the lowest measured strain amplitude, the latter, depending on the range of the experimental data, may be more difficult to determine.

The general quality of the fits based on Equation (13) is very good and not at all inferior to the Equation (5). In four out of the five examples we used one single value for $y$. The attendant values of $D / d$ are roughly between 10 and 100. We should note that both $y$ and $D / d$ may be varied in conjunction by about $\pm 20 \%$ and $\mp 50 \%$, respectively, without affecting the quality of the fits significantly. For instance, instead of $y=0.75$ and of $D / d=14$ we can equally well use $y=0.9$ and $D / d=10$. This in particular means that $y$ does not need to be constant. But it means that on the basis of the data considered here no 'fine tuning' of the parameters is sensible (aside from one example in which $y$ was found to be much greater than the standard value).

Notice that Table 1 includes additional parameter values obtained by analysing experimental storage moduli not shown in Figure 3. We shall discuss these results in more detail in the next section. However, it is worth noting already at this point that compounds containing less filler (40 and $50 \mathrm{phr}$ ) compared to compounds containing more filler $(60,70$, and $80 \mathrm{phr})$ on average yield larger ratios $D / d$. This means that the (average) gap width is greater in the lesser filled systems, which is consistent with the model, even though filler dispersion depends on many parameters of course.

The authors of the aforementioned mentioned SAXS and TEM study in reference [21] find typical aggregate dimensions of around $50 \mathrm{~nm}$. If this is our $D$, then $D / d=10$ corresponds to $d \approx 5 \mathrm{~nm}$ and $D / d=10$ to $d \approx 0.5 \mathrm{~nm}$. The former $d$-value is consistent with rubber bridges between particles discussed by some researchers in the context of the Payne effect (e.g. $[29,30])$. On the other hand, the pronounced decrease of the electrical resistance of carbon black filled elastomer compounds at the percolation threshold (e.g. [31]) indicates that there must be numerous small particle-particle separations as well - consistent with the small $d$-values quoted above. In recent work Hentschke and coworkers [32, 33] have developed a model, which explains how such narrow-gapcontacts can reversibly open due to external stress (notice that 'reversibility' here is not meant in a thermodynamic sense, because opening and closing the contact is accompanied by energy dissipation). Another indication of the importance of these narrow-gap-

Table 1. Parameter values obtained throughout this paper with the new model applied to $\mu^{\prime}(u)$ vs. $u$-data from different literature sources. $E_{\mathrm{a}}$-values were calculated only when $\mu^{\prime}(u)$ was measured for at least three different temperatures. Abbreviations: (S)SBR: (solution-polymerized) styrene butadiene rubber; A200: Aerosil ${ }^{\circledR}$ 200; U7000: Ultrasil ${ }^{\circledR} 7000$; VN2(3): Ultrasil $^{\circledR}$ VN2(3); TESPT: bis-(triethoxysilylpropyl-tetrasulfide; TESPD: bis-(triethoxysilylpropyl-disulfide; N234 and N330: carbon blacks; phr: parts [in weight] per hundred rubber.

\begin{tabular}{|l|c|c|c|l|}
\hline \multicolumn{1}{|c|}{ Reference } & $\boldsymbol{y}$ & $\boldsymbol{D} / \boldsymbol{d}$ & $\begin{array}{c}-\boldsymbol{E}_{\mathbf{a}} \\
{[\mathbf{k J} / \mathbf{m o l}]}\end{array}$ & \multicolumn{1}{|c|}{ Rubber, filler, silane } \\
\hline$[25]$ & 0.75 & 8 & 8 & SSBR, 40 phr A200, no silane \\
\hline$[26]$ (Figure 2.6) & 0.75 & 85 & - & SBR, 60 phr U7000, no silane \\
\hline$[26]$ (Figure 5.14) & 0.75 & 70 & 11 & SBR, 60 phr VN2, no silane \\
\hline$[25]$ & 0.75 & 14 & - & SSBR, 40 phr A200, 3.2 phr TESPT \\
\hline$[26]$ (Figure 5.11) & 2.80 & 9 & 26 & SBR, 60 phr U7000, TESPD \\
\hline$[28]$ & 0.75 & 4 & 21 & SSBR, 50 phr VN3, 4 phr TESPT \\
\hline$[27]$ & 0.75 & 37 & - & SSBR, 70 phr N234 \\
\hline$[26]$ (Figure 4.15) & 0.75 & 70 & 12 & Therban ${ }^{\circledR}, 80 \mathrm{phr} \mathrm{N330}$ \\
\hline
\end{tabular}


L1 contacts in the context of the Payne effect is the folL2 lowing. The onset of the Payne effect usually is obL3 served at strain amplitudes of around $0.1 \%$, i.e. $u=$ L4 0.001, which is quite small. Hence we conclude that L5 the strain amplification factor, $D / d$, introduced in

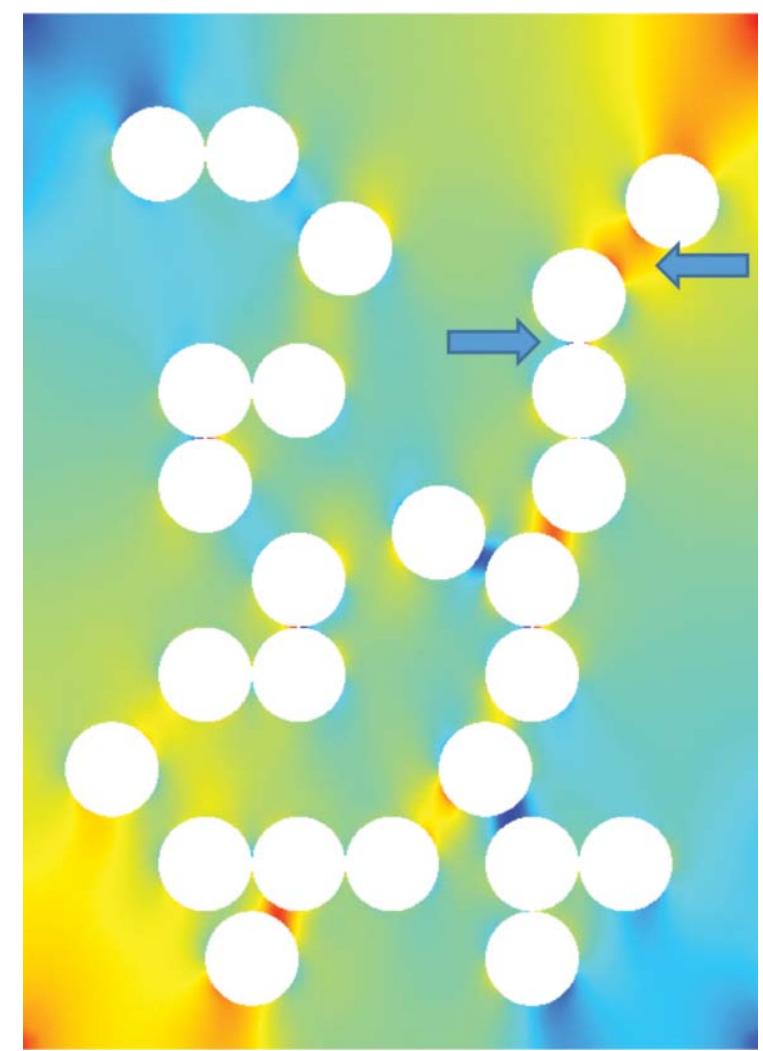

Figure 4. Normal stress calculated using the Finite Element Method in a two- dimensional system along the long direction in response to an orthogonal shear deformation (not shown explicitly). Circles indicate solid particles embedded in an elastic matrix using sticky boundary conditions. Red: large positive stress; blue: large negative stress; light green: neutral. Arrows indicate examples for the two types of gaps, i.e. wide (upper) and narrow gaps (lower arrow).

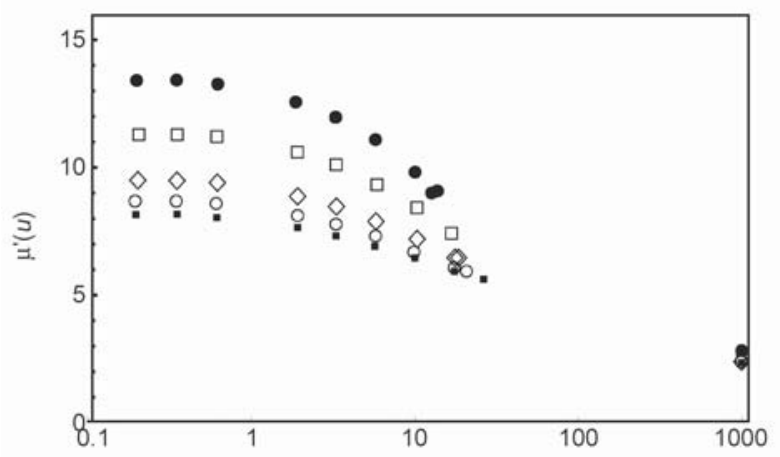

a) $u[\%]$
Equation (10), must be large, i.e. $d$ must be in the sub-nm-range, in order to yield a significant strain, $u_{\text {nano }}$ in the gap itself. In any case, we expect the above values for $D / d$ to represent averages across the system. Figure 4 is an illustration of a load-bearing filler path based on a 2D Finite Element calculation of a filled rubber. The arrows shown in this picture highlight the two types of particle-particle contacts, i.e. wide and narrow gaps.

Our above derivation of Equation (12) is quite rough. For instance, we ignore the orientation distribution of the paths relative to the applied shear. However, our estimates of the effect of orientation do not produce significantly different results. In addition, our exponent $\sigma$ is a parameter, for which we have no derivation yet. Currently work is in progress to obtain $h(L)$ from coarse-grained simulation models of filled rubbers.

\section{Temperature dependence}

The Payne effect is strongly dependent on temperature as is illustrated by the example in Figure 5a. It shows the experimental storage modulus, taken from Figure 11 in reference [25], for a series of temperatures (other analogous examples for silica and carbon black filled systems are Figure 4 in reference [28] and Figure 4.15 in reference [26], respectively.). However, changing the temperature does not affect the shape of the Payne curve significantly as is illustrated in Figure 5b, which shows the same data plotted in reduced units. To good approximation this means Equation (15):

$$
\Delta \mu^{\prime}(u, T)=\frac{\Delta \mu^{\prime}(0)}{\left(1+\frac{D}{d} u\right)^{y}} x_{\mathrm{A}}(T)
$$

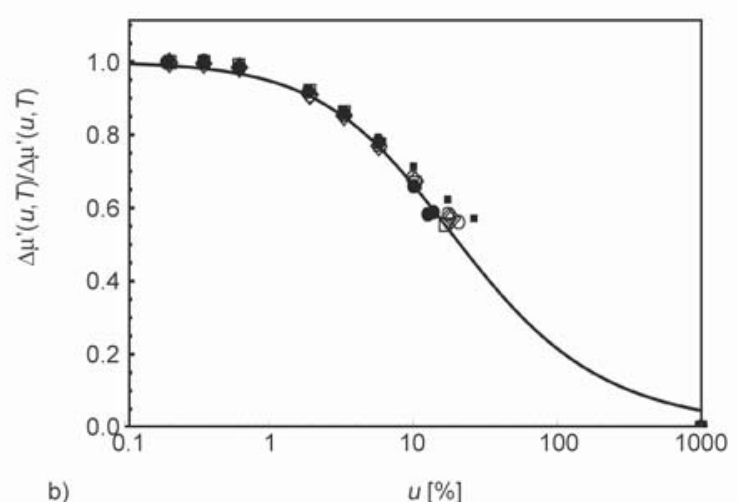

b)

$u[\%]$

Figure 5. (a) Experimental storage modulus from Figure 11 in reference [25] (S-SBR ( $50 \mathrm{wt} \%$ vinyl $/ 25 \mathrm{wt} \%$ styrene) filled with $40 \mathrm{phr}$ Aerosil ${ }^{\circledR} 200$ ). From top to bottom: $T=293,313,333,353$, and $373 \mathrm{~K}$. (b): Master plot of the data in Figure 5a. The curve is a fit based on Equation (13) with $y=0.7$ and $D / d=8$ (cf. Table 1). 
where $x_{\mathrm{A}}(T)$ is an as yet unknown function of temperature. Notice $\Delta \mu^{\prime}(0, T)=\Delta \mu^{\prime}(0) x_{\mathrm{A}}(T)$. Notice also that this $x_{\mathrm{A}}(T)$ and the quantity $x_{\mathrm{A}}$, introduced above in context of Equation (1), originally do have the same meaning. The subsequent introduction of the assumption (3), however, eliminates the connection (cf. the above discussion).

In the following we derive an expression for $x_{\mathrm{A}}(T)$. We consider a 'bond' within the contact. A bond can be a hydrogen bond between silanol groups on adjacent silica particles if $d$ is sufficiently small. It is also possible that water molecules, still residing on the silica surface, can form simultaneous hydrogen bonds with surface silanol groups on both sides of the gap [35]. A bond can also be formed by a polymer segment physisorbed on a particle surface. If the polymer itself, or as part of a network strand, bridges the gap to an adjacent filler particle then this connection can serve as a bond as well. Similar bonds can be formed involving silanols. In general, a 'bond' can be every conceivable atomic or molecular bond, which can break reversibly (in a mechanical sense) under the influence of strain (cf. Figure 6). In addition, there may be covalent particle-silane-polymersilane-particle-bonds of course. The breaking of such covalent bonds should affect the first few strain cycles only. This has been discussed by Bueche in the context

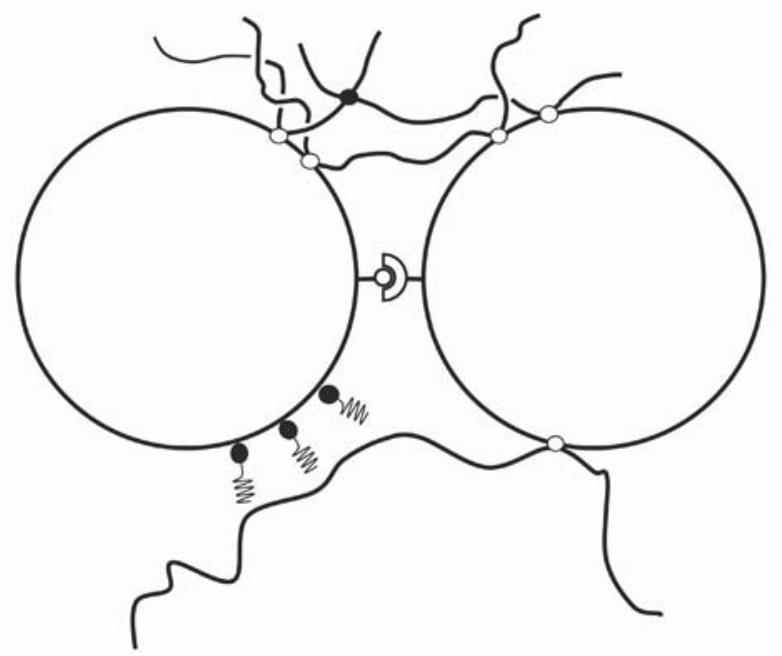

Figure 6. Different types of bonds connecting two aggregates (large circles). Top: single polymer segment or cross-linked polymer segments bridging the gap between the particles (open circles: physical bonds; closed circle: chemical bond). Centre: the symbol in the centre represents a strong direct bond (e.g. a hydrogen bridge in the case of silica). Bottom: silanes on the left aggregate prevent the physisorption of the polymer segment. of the Mullins effect [36]. The model presented here is meant to describe the subsequent behaviour.

We can describe the bond thermodynamically by the following mono-molecular reaction Equation (16):

$A \longleftrightarrow B$

$A$ is the closed bond, whereas $B$ is the open or (reversibly) broken bond. At equilibrium Equation (17) holds:

$\mu_{\mathrm{A}}(T)=\mu_{\mathrm{B}}(T)$

Here $\mu_{\mathrm{A}}$ and $\mu_{\mathrm{B}}$ are the chemical potentials of the bond in the states $A$ and $B$ (not to be confused with the moduli for which we also use the letter $\mu$ ). We neglect interactions between different bonds ('ideal gas approximation') and thus Equation (18) is obtained:

$\bar{\mu}_{\mathrm{A}}(T)+R T \ln x_{\mathrm{A}}=\bar{\mu}_{\mathrm{B}}(T)+R T \ln x_{\mathrm{B}}$

The quantities $x_{\mathrm{A}}$ and $x_{\mathrm{B}}$ are the respective mole fractions. $\bar{\mu}_{\mathrm{i}}(T)(i=A, B)$ is the chemical potential of $i$ in the hypothetical state where $x_{\mathrm{i}}=1$. As before $x_{\mathrm{A}}+x_{\mathrm{B}}=1$ and solving for $x_{\mathrm{A}}$ yields Equation (19):

$x_{\mathrm{A}}=\left(1+K^{-1}\right)^{-1}$

where $K$ is given by Equation (20):

$K=\exp \left[\frac{-\left(\bar{\mu}_{A}(T)-\bar{\mu}_{B}(T)\right)}{R T}\right]$

Additional progress is made using the GibbsHelmholtz Equation (21):

$\frac{\partial}{\partial T} \frac{\bar{\mu}_{\mathrm{i}}}{T}=-\frac{\bar{h}_{\mathrm{i}}}{T^{2}}$

where $\bar{h}_{\mathrm{i}}$ is the molar enthalpy of $\mathrm{i}$ in the state $x_{\mathrm{i}}=1$. Integration yields Equation (22):

$\frac{\bar{\mu}_{\mathrm{i}}(T)}{T}=\frac{\bar{\mu}_{\mathrm{i}}\left(T_{0}\right)}{T_{0}}-\bar{h}_{\mathrm{i}}\left(\frac{1}{T}-\frac{1}{T_{0}}\right)$

here $T_{0}$ is a reference temperature. We also assume that $\bar{h}_{\mathrm{i}}$ depends on $T$ only weakly, i.e. its temperature dependence can be neglected. Putting everything together we find Equation (23):

$x_{\mathrm{A}}=\left(1+K_{0} \exp \left[E_{\mathrm{a}}\left(\frac{1}{R T}-\frac{1}{R T_{0}}\right)\right]\right)^{-1}$

where $K_{0}$ is given by Equation (24): 
$K_{0}=\exp \left[-\frac{\bar{\mu}_{\mathrm{B}}\left(T_{0}\right)-\bar{\mu}_{\mathrm{A}}\left(T_{0}\right)}{R T_{0}}\right]$

and according to Equation (25):

$E_{\mathrm{a}} \approx \bar{h}_{\mathrm{A}}-\bar{h}_{\mathrm{B}}$

$E_{\mathrm{a}}$ is basically the energy difference between the closed and the open bond-states. Its value is characteristic for the type of bond. In the case of a silanol hydrogen bond we expect $E_{\mathrm{a}} \approx-25 \mathrm{~kJ} / \mathrm{mol}$. In the case of van der Waals interaction of a polymer segment with the particle surface we expect $E_{\mathrm{a}} \approx$ $-10 \mathrm{~kJ} / \mathrm{mol}$ or even less. If in addition we choose $T_{0}$ so that $\bar{\mu}_{\mathrm{B}}\left(T_{0}\right) \approx \bar{\mu}_{\mathrm{A}}\left(T_{0}\right)$, we can set $K_{0} \approx 1$. Our final expression for $x_{\mathrm{A}}$ therefore is Equation (26):

$x_{\mathrm{A}}(T)=\left(1+\exp \left[E_{\mathrm{a}}\left(\frac{1}{R T}-\frac{1}{R T_{0}}\right)\right]\right)^{-1}$

Figure 7 illustrates the application of Equation (26) to the data in Figure 5a, i.e. $\Delta \mu^{\prime}(u, T) \propto x_{\mathrm{A}}(T)$. The five data points correspond to the experimental $\Delta \mu^{\prime}(u, T)$ at the five different temperatures. The solid line is the best fit, whereas the broken lines are meant to show the sensitivity of the fit procedure. The 'best fit' value of the activation energy is $E_{\mathrm{a}}=-8 \mathrm{~kJ} / \mathrm{mol}$. This is in accord with our expectations for dispersive interactions (physisorption) of a small number of carbon atoms (2 to 3 ) with the particle surface. But what about other types of bonds mentioned above - for instance hydrogen bonds, which should yield more negative $E_{\mathrm{a}}$-values? We may of course argue that here $D / d=8$ ) and thus $d$ is too large to permit hydrogen bonding between the surfaces in this case. But we expect to always find a mixture of wide and narrow

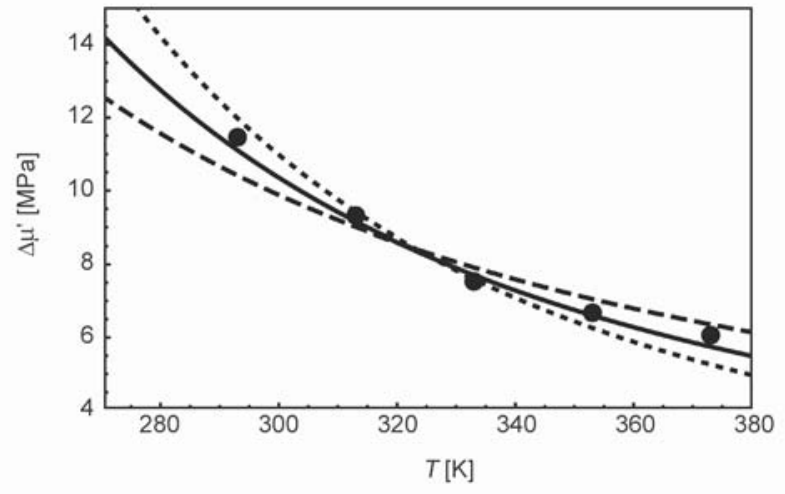

Figure 7. Fit to the data of Figure $5 \mathrm{a}$, using $\Delta \mu^{\prime}(u, T) \propto x_{\mathrm{A}}(T)$, at $u \approx 0.2 \%$ strain. Solid line: $E_{\mathrm{a}} \approx-8 \mathrm{~kJ} / \mathrm{mol}, T_{0}=$ $170 \mathrm{~K}$; dashed line: $E_{\mathrm{a}} \approx-6 \mathrm{~kJ} / \mathrm{mol}, T_{0}=147 \mathrm{~K}$; dotted line: $E_{\mathrm{a}} \approx-10 \mathrm{~kJ} / \mathrm{mol}, T_{0}=188 \mathrm{~K}$ gaps between particles. Thus, a certain fraction of the bonds should be hydrogen bonds if the filler is silica.

A second series of examples is shown in Figure 8a includes two experimental temperature series of the storage modulus, $\mu^{\prime}(u)$, vs. strain amplitude, $u$. The solid symbols are from Figure 5.11 in reference [26] corresponding from top to bottom to $T=393,418$, 438 , and $443 \mathrm{~K}$. The system is SBR filled with $60 \mathrm{phr}$ U7000 plus TESPD. The open symbols are from Figure 5.14 in reference [26] corresponding from top to bottom to $T=293,333$, and $353 \mathrm{~K}$. The system is SBR filled with $60 \mathrm{phr}$ VN2 without silane. The lines are fits using Equation (12). The values for $y$ and $D / d$ are listed in Table 1. Notice that there are only two pairs of $y$ and $D / d$, one for each system, because these numbers do not depend on temperature. Figure $8 b$ shows the fit to the first data set in Figure 8a (solid symbols) using $\Delta \mu^{\prime}(u, T) \propto x_{\mathrm{A}}(T)$, at $u \approx 0.2 \%$ strain. The solid line corresponds to $E_{\mathrm{a}} \approx-26 \mathrm{~kJ} / \mathrm{mol}$ and $T_{0}=310 \mathrm{~K}$; the meaning of the dashed and the dotted line are analogous to Figure 7. Figure 8c shows the analogous fit to the second data set in Figure 8a (open symbols). Here the solid line corresponds $E_{\mathrm{a}} \approx$ $-11 \mathrm{~kJ} / \mathrm{mol}$ and $T_{0}=194 \mathrm{~K}$. Figure $8 \mathrm{~d}$ finally shows $x_{\mathrm{A}}$ vs. $T$ according to Equation (26) (upper curve: $E_{\mathrm{a}} \approx-26 \mathrm{~kJ} / \mathrm{mol}$; lower curve: $E_{\mathrm{a}} \approx-11 \mathrm{~kJ} / \mathrm{mol}$ ).

The activation energies for these systems as well as two additional systems, whose graphs are not shown here explicitly, are compiled Table 1 . We note that the activation energies of the silanised systems are more negative than those of the non-silanised systems. Even though the system composition is not quite the same in all these cases, this still is quite striking and requires an explanation.

In order to understand this dependence of $E_{\mathrm{a}}$ on silane concentration we must extend the above approach, including more than one type of bond. We may generalize Equations (16) and (17) to Equation (27):

$$
A_{\mathrm{i}} \longleftrightarrow B_{\mathrm{i}}
$$

and Equation (28):

$$
\mu_{\mathrm{i}, \mathrm{A}}(T)=\mu_{\mathrm{i}, \mathrm{B}}(T)
$$

where $i$ denotes the different types of bonds (akin to non-reacting components; $i=1, \ldots, K)$. The total number of closed or $A$-bonds now becomes as shown by Equation (29): 

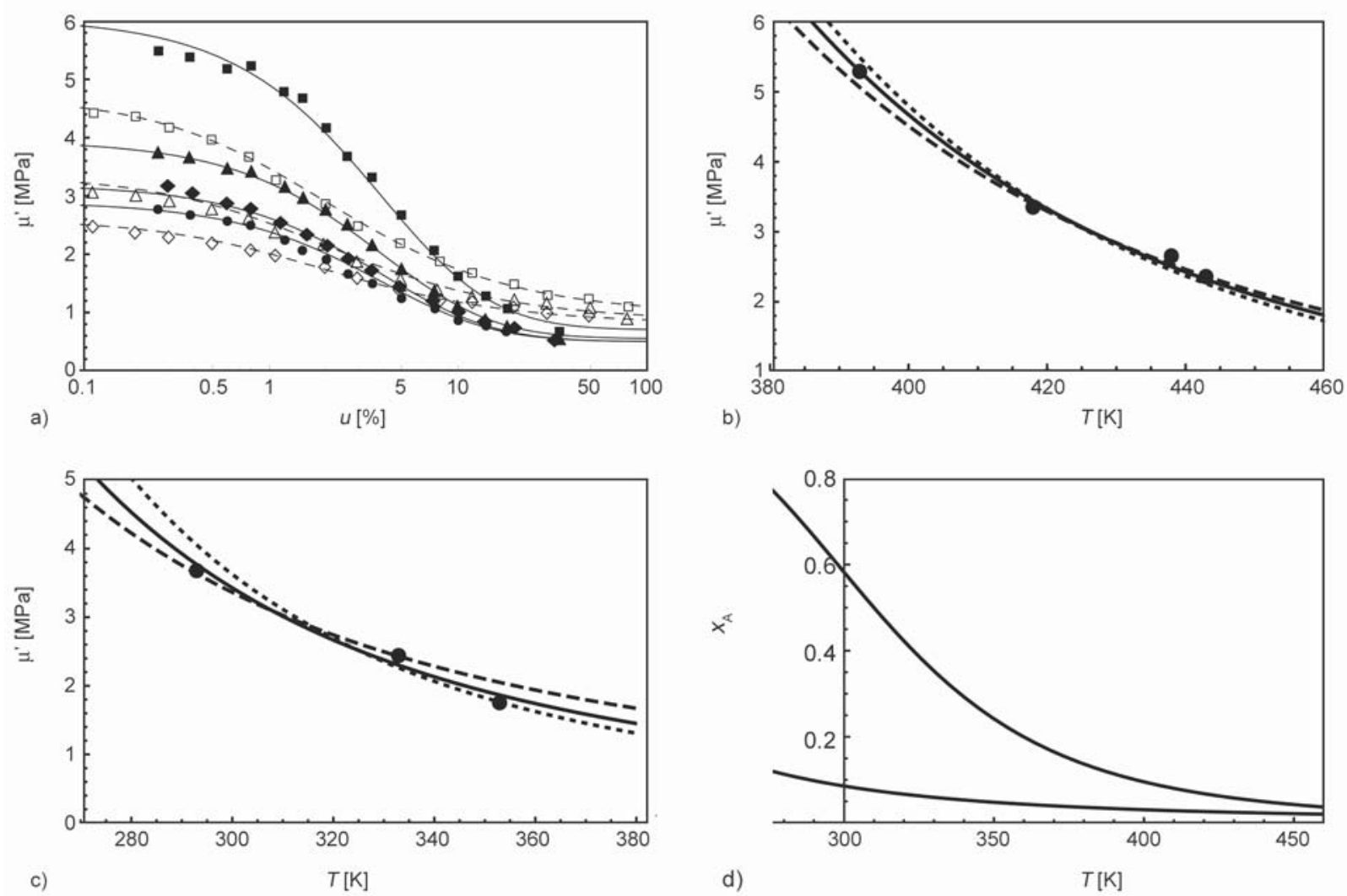

Figure 8. A second series of examples analogous to Figures 5a and Figure 7. a) Storage modulus, $\mu^{\prime}(u)$, vs. strain amplitude, $u$. Solid symbols: data from Figure 5.11 in reference [26] corresponding from top to bottom to $T=393,418,438$, and $443 \mathrm{~K}$; open symbols: data from Figure 5.14 in reference [26] corresponding from top to bottom to $T=293$, 333, and $353 \mathrm{~K}$. The lines are fits using Equation (12). b) Fit to the first data set in Figure 8a. The solid line corresponds to $E_{\mathrm{a}} \approx-26 \mathrm{~kJ} / \mathrm{mol}$ and $T_{0}=310 \mathrm{~K}$. The meaning of the dashed and the dotted line are analogous to Figure 7. c) Analogous fit to the second data set in Figure $8 \mathrm{a}$ (open symbols). Here the solid line corresponds $E_{\mathrm{a}} \approx-$ $11 \mathrm{~kJ} / \mathrm{mol}$ and $T_{0}=194 \mathrm{~K}$. d) $x_{\mathrm{A}}$ vs. $T$ according to Equation (26). Upper curve: $E_{\mathrm{a}} \approx-26 \mathrm{~kJ} / \mathrm{mol}$; lower curve: $E_{\mathrm{a}} \approx-11 \mathrm{~kJ} / \mathrm{mol}$. Details are discussed in the text.

$n_{\mathrm{A}}(T)=\sum_{\mathrm{i}=1}^{K} n_{\mathrm{i}} x_{\mathrm{i}, \mathrm{A}}(T)$

Here $n_{\mathrm{i}}$ is the molar amounts of bonds of type $i$ and $x_{\mathrm{i}, \mathrm{A}}(T)$ is the fraction of these bonds which are closed at temperature $T$. In the case $K=1$ we have $n_{\mathrm{A}}=$ $n_{1} x_{1, \mathrm{~A}}(T) \equiv n x_{\mathrm{A}}(T)$ - our previous situation. In particular Equation (15) must be replaced by Equation (30):

$\Delta \mu^{\prime}(u, T)=\frac{\Delta \mu^{\prime}(0)}{\left(1+\frac{D}{d} u\right)^{y}} \sum_{\mathrm{i}=1}^{K} x_{\mathrm{i}} x_{\mathrm{i}, \mathrm{A}}(T)$

where $x_{\mathrm{i}}=n_{\mathrm{i}} \sum_{\mathrm{i}=1}^{\mathrm{K}} n_{\mathrm{i}}$. Now we return to the above question.

We want to simplify the discussion by limiting the number of bond types to two - weak and strong, i.e. $E_{\mathrm{a} \text {,weak }}<E_{\mathrm{a} \text {,strong. }}$ Weak bonds may be inter-particle links via physisorbed (very) short polymer segments of the same polymer on both particles; an example for strong bonds are hydrogen bonds connecting the particles. Without silane we expect $n_{\text {weak }} \gg n_{\text {strong }}$. This is because polymers do possess extensive conformational freedom, which allows them access to large portions of surface area on both particles simultaneously; hydrogen bonds on the other hand are severely constrained by geometry. We therefore expect Equation (31):

$$
\begin{aligned}
n_{\mathrm{A}}(T) & =n_{\text {weak }} x_{\text {weak }, \mathrm{A}}(T)+n_{\text {strong }} x_{\text {strong,A }}(T) \approx \\
& \approx n_{\text {weak }} x_{\text {weak }, \mathrm{A}}(T)
\end{aligned}
$$

Consequently we find $E_{\mathrm{a}} \approx E_{\text {a,weak. Next we consider }}$ the opposite extreme. A large number of silanes compete with polymer segments, which are part of an interparticle link, for their adsorption sites on the particle surfaces. Because the silanes form covalent bonds with the surface, they eventually take over the aforementioned surface sites, which are then no longer available for the formation of weak bonds (cf. Figure 6). Hydrogen bonds are less affected (kinetically), 
because they are strong and not easily broken. Thus we end up with $n_{\text {weak }}$ comparable to or even smaller than $n_{\text {strong. }}$. Because in addition $x_{\text {weak,A }}(T) \ll$ $x_{\text {strong, }}(T)$, we expect to find Equation (32):

$$
\begin{aligned}
n_{\mathrm{A}}(T) & =n_{\text {weak }} x_{\text {weak }, \mathrm{A}}(T)+n_{\text {strong }} x_{\text {strong, } \mathrm{A}}(T) \approx \\
& \approx n_{\text {strong }} x_{\text {strong, } \mathrm{A}}(T)
\end{aligned}
$$

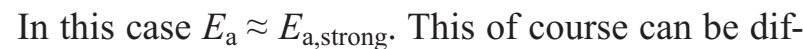
ferent if the silanes offset the weak bond reduction by contributing bonds of their own, either by physisorption of alkyl moieties on the surface of the opposite particle or via new load-bearing paths due to silane-polymer-silane coupling of particles. However, currently the author is not aware of such an example.

We want to point out that the above reasoning can be used to compare the number of weak to the number of strong sites. This is because, as shown by Equation (33):

$$
\begin{aligned}
& \frac{\Delta \mu^{\prime}(\text { no silane, } T, u \approx 0)}{\Delta \mu^{\prime}(\text { silane saturation, } T, u \approx 0)} \approx \\
& \approx \frac{n_{\text {weak }} x_{\text {weak, } \mathrm{A}}(T)}{n_{\text {strong }} x_{\text {strong, }}{ }^{(T)}}
\end{aligned}
$$

here $x_{\ldots, \mathrm{A}}(T)$ can be calculated from Equation (26). Finally, it is worth mentioning that the above reference temperatures, $T_{0}$, and their attendant activation energies, $E_{\mathrm{a}}$, apparently are not independent. Figure 9 shows a linear fit to the $E_{\mathrm{a}}-T_{0}$-pairs for which $E_{\mathrm{a}}$ is listed in Table 1 . There is one stray value at $-12 \mathrm{~kJ} / \mathrm{mol}$ and $250 \mathrm{~K}$. Notice that the attendant system differs in both its polymer and filler type from the other systems. The data base is limited of course, forbidding further elaboration of this point.

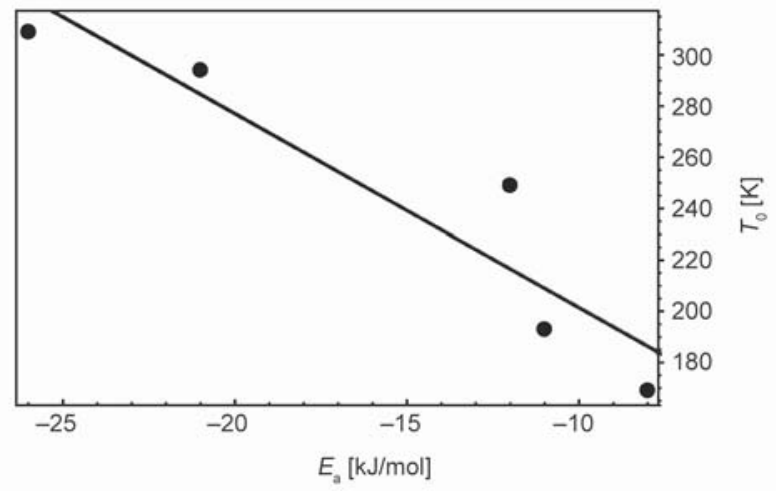

Figure 9. Temperatures, $T_{0}$, vs. activation energies, $E_{\mathrm{a}}$, for the values listed in Table 1. The straight line fit is $T_{0}=126 \mathrm{~K}-7.6 E_{\mathrm{a}} \mathrm{mol} \cdot \mathrm{K} / \mathrm{kJ}$.
It is important to note that the application of different activation energies to this context is not new. The authors of references [12] and [26] distinguish between strongly and weakly adsorbed chain segments and their relative abundance. However, the energy site distributions measured on the surfaces of commercial filler particles (cf. [37] for carbon blacks or [38] for silicas) are broad and quite unspecific. The rather clear distinction between activation energies of small magnitude, typical for dispersion forces, and those of large magnitude, typical for hydrogen bonds, is a strong indication that chain slippage, as advocated in the aforementioned references, is not sufficient to explain the Payne effect - at least in silica filled systems.

\section{Discussion of the model in relation to key observations}

Filled rubber compounds are truly complex materials. Nevertheless, there are certain general observations related to the Payne effect an attendant model should not be in contradiction to and at least be able to explain qualitatively. The following is a concise compilation of such observations and their relation to the present model. (i) First there is the decrease of the Payne curve with increasing temperature of which Figure 5a is an example. The present model can explain this general observation as discussed in detail in the previous section. (ii) When the filler is silica and a covering agent (e.g. alkyl-silane) is added, then the Payne curve is lowered as well (e.g. Figure 18 in reference [22]). Addition of a covering agent or compatibiliser will affect the dispersion of the filler, which in the present model affects the exponent $y$, but it also reduces the number of bonds as discussed in the context of Equations (31) and (32). This weakens the contacts thereby reducing $\Delta \mu^{\prime}(0, T)$. (iii) Increasing the spacer length of an alkyl-silane also tends to reduce $\Delta \mu^{\prime}(0, T)$. In the context of the present model an increase of the spacer length primarily reduces the number of available surface sites (on the particle to which the silane is chemically bonded), which, at least in part, will be occupied by the spacer. (iv) The Payne curve of the uncured compounds in comparison to the cured compound is lower, i.e. $\Delta \mu_{\text {uncured }}^{\prime}(0, T)<\Delta \mu_{\text {cured }}^{\prime}(0, T)$ (e.g. Figure 7 in reference [39]). Curing creates chemical crosslinks, which, as we have explained in the context of Figure 6, tend to increase the bonding of aggregates forming a contact. (v) Decreasing the filler content 
also reduces $\Delta \mu^{\prime}(0, T)$ (e.g. Figure 4 in reference [22]). Here we refer to the range of filler concentrations close to and above the percolation threshold. We had briefly mentioned above that this can be understood on the basis of 'fractal filler flocs' (cf. reference [5]; see also chapter 7 in reference [40]). But aside from the functional dependence of $\Delta \mu^{\prime}(0, T)$ on filler volume fraction, a reduction of filler always reduces the number of bonds with the polymer matrix. This in turn reduces the force coupling between aggregates forming a contact. (vi) Finally, and as described already, the (average) activation energy appears to increase upon increase of silane concentration. Thus, the present model, of which the aggregate-to-aggregate contact, in terms of different types of bonds across a gap, is the central element, appears to be in accord with the different general observations relating to the Payne effect.

\section{Dynamic loss}

In a typical rheological experiment, in the context of mechanical rubber testing, a disk-shaped biconical specimen is subjected to a torsion $\varphi(t)$ via the lower die of the sample chamber, whereas the attendant torque $N(t)$, transmitted through the sample to the upper die of the sample chamber, is measured with high precision. The biconical design of the sample chamber maximises spatial homogeneity of the strain. Usually $\varphi(t)=\varphi_{0} \sin (\omega t)$, where $\omega$ is a certain frequency and $t$ is time. The area enclosed by the resulting curve in the $\varphi(t)-N(t)$-plane is the dissipated energy or loss in the sample, $W_{\text {tot }}$, (at constant temperature). For an isotropic linear elastic specimen the shear modulus, $\mu$, is given by $\mu=(3 / 2) N_{0}\left[\left(\varphi_{0} \theta\right) \pi R^{3}\right]$, where $N_{0}$ is the torque amplitude, $\theta$ is twice the cone angle of the sample chamber, and $R$ is its radius. In a linear viscoelastic material this formula can be used for both the storage modulus, $\mu^{\prime}$, and the loss modulus, $\mu^{\prime \prime}$, when in the former case $N_{0}$ is replaced by the $N_{0} \cos \delta$ and in the latter it is replaced by $N_{0} \sin \delta$. Here $\delta$ is the phase angle between torsion and torque. In particular, the dissipated energy per volume, $w$, is given by $w \propto \mu^{\prime \prime}\left(\varphi_{0} \theta\right)^{2}$, where $\varphi_{0} \theta$ is the strain amplitude, $u$. In a non-linear viscoelastic material, i.e. in the context of the Payne effect, $\mu^{\prime \prime}$, akin to $\mu^{\prime}$, does depend on $u$. Nevertheless, most results for $\mu^{\prime \prime}$ vs. $u$ (as well as $\mu^{\prime}$ vs. $u$ ) are based on analysing (or fitting) the now distorted curve in the $\varphi(t)-N(t)$-plane via the expressions of linear viscoelastic theory. We find this

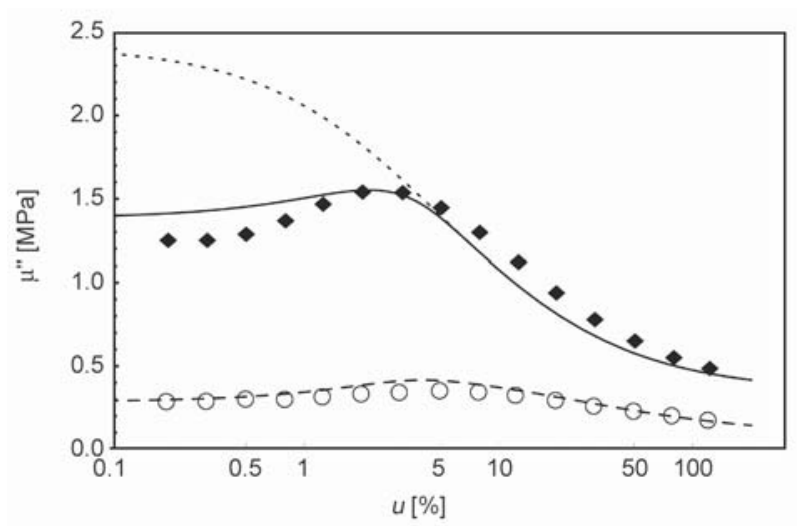

Figure 10. Strain dependence of the loss modulus, $\mu^{\prime \prime}$. Symbols: experimental loss modulus at $70^{\circ} \mathrm{C}$ and $10 \mathrm{~Hz}$ for SSBR compounds with different loading of carbon black N234 (diamonds: $70 \mathrm{phr}$; circles: $40 \mathrm{phr}$ ) taken from Figure 6 of reference [27]. Lines: different results of the model as explained in the text.

exposition important in this context, because it affects the interpretation of experimental data. For instance, the conversion from measured dissipated energy to loss modulus is strongly affected by the factor $u^{-2}$ physically motivated only in the linear theory.

Figure 10 shows experimental data for the loss modulus vs. strain amplitude taken from reference [27]. The systems are SSBR filled with $70 \mathrm{phr}$ (diamonds) and $40 \mathrm{phr}$ (circles) $\mathrm{N} 234\left(\omega=10 \mathrm{~Hz}, T=70^{\circ} \mathrm{C}\right)$, respectively. The storage modulus in the case of the diamond data is included among the data sets depicted in Figure 3. The loss modulus of the corresponding unfilled system (not shown here) is about an order of magnitude lower. This is typical at sufficiently high temperatures. Thus we may conclude that the dominant mechanisms causing the loss to occur in the filled system are tied to the filler surfaces when the temperature is high. Here and in the following we presume that in this temperature range, which is important for controlling the rolling resistance, the dominant amplitude dependent loss mechanism is associated with the reversible opening of narrowgap-contacts between filler aggregates under strain. In two previous references [32, 33] we have developed a model describing the energy dissipated by a single narrow-gap-contact, $W$, according to Equation (34):

$W=\oint \mathrm{d} x_{0} f\left(x_{0}, D, u(\omega)\right)$

where $f$ is given by Equation (35): 


$$
\begin{aligned}
f & =f_{\mathrm{pp}, 0}\left(x_{0}, D\right)+f_{\mathrm{pp}, 1}\left(x_{0}, D\right) n_{\mathrm{A}}(T)+ \\
& +f_{\text {matrix }}\left(x_{0}, D, u(\omega)\right)
\end{aligned}
$$

when it is reversibly opened and closed due to an external force. Here $x_{0}$, which is equal to the above gapwidth, $d$, at vanishing strain, is integrated over one strain cycle and $f$ is the total force between the two aggregates of a contact. The reason why the integral in Equation (34) can be different from zero is that $x_{0}=x_{0}(f)$ usually is a multi-valued function, i.e. $f$ differs during opening and closing of the contact. The underlying mechanism was discussed first in reference [32]. Subsequently, in reference [33], a combination of molecular modelling, to obtain the force contributions $f_{\mathrm{pp}, 0}\left(x_{0}, D\right)$ and $f_{\mathrm{pp}, 1}\left(x_{0}, D\right)$, and continuum theory, to describe $f_{\text {matrix }}\left(x_{0}, D, \mu(\omega)\right)$, was used to estimate in particular the dependence of the loss modulus in silica filled rubbers containing variable amount of alkyl-silanes. Notice that $f_{\mathrm{pp}, 0}\left(x_{0}, D\right)$ accounts for the temperature independent (or at least almost temperature independent) part of the particleparticle interaction, i.e. dispersion interactions. This contribution, however, is significantly smaller than $f_{\mathrm{pp}, 1}\left(x_{0}, D\right) \cdot f_{\mathrm{pp}, 1}\left(x_{0}, D\right)$, which here is multiplied by $n_{\mathrm{A}}(t)$ of Equation (29), is due to molecular bonds between the particle surfaces as outlined in Figure 6. It is worth noting that a factor $f_{\mathrm{pp}, 1}\left(x_{0}, D\right)$, is absorbed in $\Delta \mu^{\prime \prime}(0)$ in Equation (30) as well. Finally, $f_{\text {matrix }}$ accounts for the (visco-)elastic restoring force exerted by the surrounding polymer matrix on the aggregates in the contact when the latter is opened. Notice that the $\mu(\omega)$ is the matrix shear modulus and $\omega$ is the strain frequency. For instance, increasing the matrix shear modulus, for example by increasing the sulphur content, in this model reduces $W$ and therefore the loss.

What the model for $W$ does not describe, however, is the strain amplitude dependence of the total dissipated energy, as given by Equation (36):

$W_{\mathrm{tot}}(u)=W M(u)$

where $M(u)$ is the sum of all contributing contacts. Notice that $M(u)$ is dependent on the filler volume fraction, $\phi$, (not to be confused with the torsion angle introduced above). In the spirit of the above scaling approach we expect $M(u) \propto \phi^{\mathrm{y}^{\prime}}$, where $y^{\prime}$ usually is between 3 to 4 (cf. reference [34] and the discussion therein; notice that the same factor also is included in $\Delta \mu^{\prime}(0)$ in Equation (30).). In the following we focus on the high temperature regime, i.e. the regime associated with rolling resistance. Neglecting the bulk-polymer contribution we describe the amplitude dependence of the loss modulus via Equation (37):

$\mu^{\prime \prime}(u) \propto \frac{W_{t o t}(u)}{u^{2}}+\mathrm{const}=\frac{W M(u)}{u^{2}}+\mathrm{const}$

where const stands for filler induced amplitude-independent contributions to the loss modulus. In the following we apply the current model to the calculation of the u-dependence of $M(u)$.

The idea is that the number of load-bearing paths is proportional to the number of contacts in these paths which will open when the paths are strained, as shown by Equation (38):

$$
\begin{aligned}
M(u) & \propto \int_{0}^{u} \mathrm{~d} u^{\prime}\left(1-\frac{1}{\left(1+\frac{D}{d} u\right)^{y}}\right)= \\
& =u+\frac{\left(1+\frac{D}{d} u\right)^{1-y}-1}{\frac{D}{d}(y-1)} \approx \\
& \approx\left\{\begin{array}{cc}
\frac{1}{2} \frac{D}{d} y u^{2} & u \text { small } \\
u & u \text { large and } y>0
\end{array}\right.
\end{aligned}
$$

Notice that the integrand is one minus the amplitude dependent part of the distribution function $h\left(L^{\prime}\right)$, including the amplitude amplification discussed in the context of Equation (10). We must integrate over all contacts opened during the increase of the strain from 0 to its final value, $u$. The dotted line in Figure 10 corresponds to a rough fit to the data based on the relations (37) and (38), where $W$ and const are adjustable parameters. The quantities $D / d$ and $y$, on the other hand, are fixed. They are the same as before in the case of the storage modulus (cf. Table 1). The perhaps most notable discrepancy is the absence of the maximum exhibited by the experimental data.

What can be the cause of the depression of $\mu^{\prime \prime}(u)$ at small strain amplitudes? Even though $u$ is an external strain, it has a local effect on all contacts throughout the system. Generally $u$ can be viewed as as an average strain acting on each contact as discussed above in the context of $u_{\text {nano. }}$. However, there are always spatial fluctuations or inhomogeneities, which means that locally the strain can be somewhat larger or smaller compared to the average strain. Thus instead of (38) we introduce Equation (39):

$$
M(u) \propto \int_{0}^{u} \mathrm{~d} u^{\prime} \int_{0}^{\infty} \mathrm{d} \delta u f\left(\delta u, u^{\prime}\right)\left(1-\frac{1}{\left(1+\frac{D}{d} u^{\prime}\right)^{y}}\right)
$$


The weighting function $f(\delta u, u)$, approximated by Equation (40):

$$
f(\delta u, u)=\exp \left[-\frac{1}{2}\left(\frac{\delta u-u}{s}\right)^{2}\right]
$$

permits regions with slightly lower or larger local strain amplitude, $u$, to contribute to the energy dissipation from opened contacts. However, the dominant regions are those whose local strain is close to the average strain. We can evaluate the double-integral in (39) numerically, but a simple approximation is more convenient to use. Notice that the contribution of $f(\delta u, u)$, is quite independent of the value of $u$ when $u$ is larger than $s$, i.e. $u$ exceeds the width of $f(\delta u, u)$. On the other hand, for small $u$ the term $\left(1+(D / d) u^{\prime}\right)^{-y}$ varies slowly and $f\left(\delta u, u^{\prime}\right)$ can be approximated by $f(\delta u, u)$. Thus Equation (41) is obtained:

$$
\begin{aligned}
& \int_{0}^{u} \mathrm{~d} u^{\prime} \int_{0}^{\infty} \mathrm{d} \delta u f\left(\delta u, u^{\prime}\right)\left(1-\frac{1}{\left(1+\frac{D}{d} u^{\prime}\right)^{\mathrm{y}}}\right) \approx \\
& \approx \int_{0}^{\infty} \mathrm{d} \delta u f(\delta u, u) \int_{0}^{u} \mathrm{~d} u^{\prime}\left(1-\frac{1}{\left(1+\frac{D}{d} u^{\prime}\right)^{\mathrm{y}}}\right)
\end{aligned}
$$

Checking this approximation numerically we find, as expected, that for $u>s$ the deviation between the exact result and the approximation is negligible, whereas for $u<s$ the maximum deviation is about $10 \%$. If Equation (39) is evaluated via (41) we find Equation (42):

$$
\begin{array}{r}
\mu^{\prime \prime}(u) \propto W \frac{1}{u^{2}} \sqrt{\frac{\pi s^{2}}{2}}\left(1+\operatorname{erf}\left[\frac{u}{\sqrt{2} s}\right]\right) . \\
\left(u+\frac{\left(1+\frac{D}{d} u\right)^{1-y}-1}{\frac{D}{d}(y-1)}\right)+\text { cons } 1
\end{array}
$$

This expression yields the solid line in Figure 10. Here we set $s=0.022$, whereas the values for $D / d$ and $y$ remain those listed in Table 1.

All in all this means that at every strain amplitude, $u$, there are dissipation contributions from regions inside the system in which the local strain is $u \pm 2.2 \%$. This does not affect $\mu^{\prime \prime}$ at large amplitudes. The situation is different, however, when $u$ is smaller than the width of $f(\delta u, u)$. Now the contributions to the loss from regions with smaller strain than the average strain are cut off due to the lower limit in the $\delta u$-integral (notice: $\delta u>0$ ). This decreases the overall number of contacts contributing to the total dissipated energy and thus reduces $\mu^{\prime \prime}$. It is worth emphasizing the real physical nature of the phenomenon, rather than this being a mere mathematical 'trick'.

Figure 10 also includes a dashed line. The only change compared to the solid line, aside from the adjustment of an overall scale factor and a different offset, due to the very different filler content, is the reduction of $D / \mathbb{d}$ from 37, the value from Table 1, to 7 . Previously we have argued that a reduction of the filler content should result in a decrease of $D / d$, because less filler is expected to increase the number of wider contacts, i.e. contacts with smaller ratio $D / d$. Here we observe that this shifts the maximum of $\mu^{\prime \prime}(u)$ to slightly larger amplitudes, while the maximum itself becomes less pronounced. Qualitatively the same is observed in experimental systems as exemplified by the circle data. This is an interesting and non-trivial feature of the current model, which lends additional support to the above line of reasoning. Ulmer, in an overview article [41] on the strain dependence of dynamic mechanical properties of carbon black-filled rubber compounds, has introduced an additive exponential $\sim \exp \left[(-u) / u_{2}\right]$, where $u_{2}$ is an adjustable parameter, to account for the low amplitude depression of the loss modulus. This addition is purely empirical, whereas the above weighting function has a physical meaning related to the strain distribution in the filler network at a given strain amplitude. On the other hand, the above Gaussian is a mere plausible assumption regarding the shape of the distribution. It certainly is desirable to find a more stringent means to obtain this function. The experimental evidence (e.g. Figure 11 in the reference [41]) points towards a possibly more complicated shape to account for the apparent, albeit small, oscillations in the loss modulus at very small strain. Finally, it is worth noting that a (pronounced) maximum of $\mu^{\prime \prime}(u)$ is not always observed. This may be because of a much smaller s, corresponding to greater uniformity in the system, which we expect to vary depending on processing and testing conditions, or simply because no data were obtained at sufficiently small $u$.

\section{Conclusions}

In this work we develop a new model replacing the form $\mu^{\prime}(u)-\mu^{\prime}(\infty) \propto\left(1-\left(u / u_{0}\right)^{2 \mathrm{~m}}\right)^{-1}$ with a modified expression based on a scaling approach. The parameter $u_{0}$ is expressed in terms of the aggregate size and an effective inter-particle spacing along the loadbearing network strands. The exponent is related to the fractal dimension of the filler network and the 
attendant self-similar distribution of minimum path lengths. The new function offers additional inside as to the microscopic cause of the Payne effect. We also develop a theoretical approach to the temperature dependence of the Payne effect and how it relates to the chemical composition of the inter-particle interfaces. The different activation energies found experimentally yield rather strong evidence for different types of aggregate-to-aggregate bonding, which include previous ideas regarding the amplitude dependent detachment of chain segments, rubber-bridges as well as strong bonds requiring the aggregates to be in close contact. Using the approach developed here it should be possible to compare the relative number of different bonding sites available for instance at variable silane coverage. Finally, the model is extended to describe the attendant amplitude dependence of the loss modulus. All in all, the set of equations, Equation (30) together with Equations (26) and (42) together with Equation (34), approximate the contributions of the filler network, based on the aggregate-to-aggregate contact as the basic building block, to the storage and the loss modulus, respectively, in filled rubber networks.

\section{Acknowledgements}

The author wishes to thank Ralf Bäuerlein, MonTech (Rubber Testing Solutions), for the e-mail exchange regarding the measurement of dynamic moduli and Jan Meyer for his valuable comments and suggestions.

\section{References}

[1] Stambaugh R. B.: Vibration properties of rubberlike materials. Industrial and Engineering Chemistry, 34, 1358-1365 (1942). https://doi.org/10.1021/ie50395a024

[2] Payne A. R.: Dynamic properties of filler-loaded rubbers. in 'Reinforcement of elastomers' (ed.: Kraus G.) Interscience Publishers, New York, 69-123 (1965).

[3] Ouyang G. B.: Modulus, hysteresis and the Payne effect. Network junction model for carbon black reinforcement. Kautschuk Gummi Kunststoffe, 59, 332-342 (2006).

[4] Ouyang G. B.: Network junction model for carbon black reinforcement. The Payne effect. Part II. Kautschuk Gummi Kunststoffe, 59, 454-458 (2006).

[5] Vilgis T. A., Heinrich G., Klüppel M.: Reinforcement of polymer nano-composites: Theory, experiments and applications. Cambridge University Press, New York (2009).
[6] Kraus G.: Reinforcement of elastomers. Wiley, New York (1965).

[7] Kraus G.: Mechanical losses in carbon-black-filled rubbers. Applied Polymer Symposia, 39, 75-92 (1984).

[8] Böhm G. G. A., Nguyen M. N.: Flocculation of carbon black in filled rubber compounds. I. Flocculation occurring in unvulcanized compounds during annealing at elevated temperatures. Journal of Applied Polymer Science, 55, 1041-1050 (1995).

https://doi.org/10.1002/app.1995.070550707

[9] Heinrich G., Klüppel M.: Recent advances in the theory of filler networking in elastomers. Filled Elastomers Drug Delivery Systems, 160, 1-44 (2002).

https://doi.org/10.1007/3-540-45362-8 1

[10] Witten T. A., Rubinstein M., Colby R. H.: Reinforcement of rubber by fractal aggregates. Journal de Physique II France, 3, 367-383 (1993).

https://doi.org/10.1051/jp2:1993138

[11] Huber G., Vilgis T. A.: Universal properties of filled rubbers: Mechanisms for reinforcement on different length scales. Kautschuk Gummi Kunststoffe, 52, 102107 (1999).

[12] Maier P., Göritz D.: Molecular interpretation of the Payne effect. Kautschuk Gummi Kunststoffe, 49, 1821 (1996).

[13] Sternstein S. S., Zhu A-J.: Reinforcement mechanism of nanofilled polymer melts as elucidated by nonlinear viscoelastic behavior. Macromolecules, 35, 7262-7273 (2002).

https://doi.org/10.1021/ma020482u

[14] Montes H., Lequeux F., Berriot J.: Influence of the glass transition temperature gradient on the nonlinear viscoelastic behavior in reinforced elastomers. Macromolecules, 36, 8107-8118 (2003).

https://doi.org/10.1021/ma0344590

[15] Heinrich G., Vilgis T. A.: A statistical mechanical approach to the Payne effect in filled rubbers. Express Polymer Letters, 9, 291-299 (2015). https://doi.org/10.3144/expresspolymlett.2015.26

[16] Kaliske M., Rothert H.: Constitutive approach to rateindependent properties of filled elastomers. International Journal of Solids and Structures, 35, 2057-2071 (1998). https://doi.org/10.1016/S0020-7683(97)00182-0

[17] Lion A.: Phenomenological modelling of strain-induced structural changes in filler-reinforced elastomers. Kautschuk Gummi Kunststoffe, 58, 157-162 (2005).

[18] Gil-Negrete N., Viñolas J., Kari L.: A simplified methodology to predict the dynamic stiffness of carbon-black filled rubber isolators using a finite element code. Journal of Sound and Vibration, 296, 757-776 (2006). https://doi.org/10.1016/j.jsv.2006.03.038

[19] Höfer P., Lion A.: Experimental investigations on and modelling of the transient phenomena of the Payne-effect. Proceedings in Applied Mathematics and Mechanics, 8, 10425-10426 (2008). https://doi.org/10.1002/pamm.200810425 
[20] Rendek M., Lion A.: Amplitude dependence of fillerreinforced rubber: Experiments, constitutive modelling and FEM - Implementation. International Journal of Solids and Structures, 47, 2918-2936 (2010). https://doi.org/10.1016/j.ijsolstr.2010.06.021

[21] Baeza G. P., Genix A-C., Degrandcourt C., Petitjean L., Gummel J., Couty M., Oberdisse J.: Multiscale filler structure in simplified industrial nanocomposite silica/ SBR systems studied by SAXS and TEM. Macromolecules, 46, 317-329 (2013).

https://doi.org/10.1021/ma302248p

[22] Luginsland H-D.: A review on the chemistry and the reinforcement of the silica-silane filler system for rubber applications. Shaker Verlag, Aachen (2002).

[23] Hentschke R.: Thermodynamics. Springer, Heidelberg (2014).

[24] de Gennes P-G.: Scaling concepts in polymer physics. Cornell University Press, Ithaca (1979).

[25] Stöckelhuber K. W., Svistkov A. S., Pelevin A. G., Heinrich G.: Impact of filler surface modification on large scale mechanics of styrene butadiene/silica rubber composites. Macromolecules, 44, 4366-4381 (2011). https://doi.org/10.1021/ma1026077

[26] Böhm J.: Der Payneeffekt: Interpretation und Anwendung in einem neuen Materialgesetz für Elastomere. Ph.D. Thesis, Universität Regensburg, Regensburg (2001).

[27] Wang M-J.: The role of filler networking in dynamic properties of filled rubber. in 'Proceedings of the meeting of the American Chemical Society Rubber Division. Indianapolis, Indiana' 48/1-48/35 (1998).

[28] Qu L., Wang L., Xie X., Yu G., Bu S.: Contribution of silica-rubber interactions on the viscoelastic behaviors of modified solution polymerized styrene butadiene rubbers (M-S-SBRs) filled with silica. RSC Advances, 4, 64354-64363 (2014).

https://doi.org/10.1039/C4RA09492A

[29] Merabia S., Sotta P., Long D. R.: A microscopic model for the reinforcement and the nonlinear behavior of filled elastomers and thermoplastic elastomers (Payne and Mullins effects). Macromolecules, 41, 8252-8266 (2008).

https://doi.org/10.1021/ma8014728

[30] Guy L., Daudey S., Cochet P., Bomal Y.: New insights in the dynamic properties of precipitated silica filled rubber using a new high surface silica. Kautschuk Gummi Kunststoffe, 62, 383-391 (2009).
[31] Niedermeier W., Fröhlich J., Luginsland H-D.: Reinforcement mechanism in the rubber matrix by active fillers. Kautschuk und Gummi Kunststoffe, 55, 356366 (2002).

[32] Hentschke R.: A nano-mechanical model for energy dissipation in filler networks. in 'Constitutive models for rubber VIII' (eds.: Gil-Negrete N., Alonso A.) CRC Press, Leiden, 299-314 (2013).

[33] Hentschke R., Hager J., Hojdis N. W.: Molecular modeling approach to the prediction of mechanical properties of silica-reinforced rubbers. Journal of Applied Polymer Science, 131, 40806/1-40806/9 (2014).

https://doi.org/10.1002/app.40806

[34] Xi H., Hentschke R.: The influence of structure on mechanical properties of filler networks via coarse-grained modeling. Macromolecular Theory and Simulation, 23, 373-382 (2014). https://doi.org/10.1002/mats.201400009

[35] Hentschke R., Ballnus C., Meyer J.: Remarks on the interpretation of IR-absorption studies applied to the surfaces of silica nanoparticles. The Journal of Physical Chemistry C, 120, 9229-9235 (2016). https://doi.org/10.1021/acs.jpcc.6b00807

[36] Bueche F.: Molecular basis for the Mullins effect. Journal of Applied Polymer Science, 4, 107-114 (1960). https://doi.org/10.1002/app.1960.070041017

[37] Schröder A.: Charakterisierung verschiedener Russtypen durch systematische statische Gasadsorption; energetische Heterogenität und Fraktalität der Partikeloberflähe. PhD thesis, Universität Hannover (2000).

[38] Wang M. J., Wolff S., Donnet J-B.: Filler-elastomer interactions. Part 2: Investigation of the energetic heterogeneity of silica surfaces. Kautschuk Gummi Kunststoffe, 45, 11-17 (1992).

[39] Luginsland H-D., Fröchlich J., Wehmeier A.: Influence of different silanes on the reinforcement of silica-filled rubber compounds. Rubber Chemistry and Technology, 75, 563-579 (2002). https://doi.org/10.5254/1.3544984

[40] Marangoni A. G., Wesdorp L. H.: Structure and properties of fat crystal networks. CRC Press, Boca Raton (2013).

[41] Ulmer J. D.: Strain dependence of dynamic mechanical properties of carbon black-filled rubber compounds. Rubber Chemistry and Technology, 69, 15-47 (1996). https://doi.org/10.5254/1.3538354 